\title{
APLICAÇÃO DE PROTOCOLO DE PREVENÇÃO DE ÚLCERA POR PRESSÃO NO CONTEXTO DOMICILIAR: UMA TRAJETÓRIA PERCORRIDA*
}

Geridice Lorna Andrade de Moraes¹, Cíntia Lira Borges², Edmara Teixeira Oliveira ${ }^{3}$, Luana Rodrigues Sarmento4, Patrícia Rebouças Araújo ${ }^{5}$, Maria Josefina da Silva ${ }^{6}$

RESUMO: A prevenção de úlceras por pressão é mais eficaz e economicamente viável que seu tratamento, para tal há protocolos padronizados e normativos. O objetivo deste estudo foi verificar a influência da aplicação de um protocolo de enfermagem para a prevenção de úlceras por pressão em idoso acamado no domicílio. Esta pesquisa dividiu-se em duas etapas: a validação de conteúdo por enfermeiras especialistas em estomaterapia e a validação clínica com pacientes idosos com risco de desenvolver esses agravos. Foi observado que o protocolo é válido e tem confiabilidade atestada nos aspectos revelados mais importantes para a população idosa que apresenta risco para adquirir úlcera por pressão.

DESCRITORES: Protocolos de enfermagem; Úlcera por pressão; Assistência domiciliar.

\section{APPLICATION OF A PROTOCOL FOR PREVENTION OF PRESSURE ULCERS IN THE HOME CONTEXT: A TRAJECTORY COVERED}

\begin{abstract}
The prevention of pressure ulcers is more efficient and economically viable than their treatment; hence there are standardized and normative protocols. This study's objective was to ascertain the influence of the application of a nursing protocol for the prevention of pressure ulcers in bed-bound older adults at home. This research was divided in two stages: the validation of content by specialist stomatherapy nurses and the clinical validation with elderly patients at risk of developing these risks to health. It was observed that the protocol is valid and that its reliability is attested in the aspects shown to be most important for the elderly population which is at risk for acquiring pressure ulcers.

DESCRIPTORS: Nursing protocols; Pressure ulcers; Home care.

\section{APLICACIÓN DE PROTOCOLO DE PREVENCIÓN DE ÚLCERA POR PRESIÓN EN EL CONTEXTO DOMICILIAR: UNA TRAYECTORIA RECORRIDA}

RESUMEN: La prevención de úlceras por presión es más eficaz y economicamente viable que su tratamiento. Para eso, hay protocolos tipificados y normativos. El objetivo de este estudio fue verificar la influencia de la aplicación de un protocolo de enfermería para la prevención de úlceras por presión en anciano encamado en domicilio. Esta investigación se ha organizado en dos etapas: la validación de contenido por enfermeras especialistas en estomaterapia y la validación clínica con pacientes ancianos con riesgo de desarrollar eses agravios. Se observó que el protocolo es válido y tiene confiabilidad testificada en los aspectos revelados más importantes para la población anciana que presenta riesgo de adquirir úlcera por presión.

DESCRIPTORES: Protocolos de enfermería; Úlcera por presión; Asistencia domiciliar.

*Extraído da tese 'Adaptação e validação de protocolo para prevenção de úlcera por pressão em idosos assistidos no domicílio' apresentada ao Programa de Pós-Graduação em Enfermagem da Universidade Federal do Ceará - UFC, em 2011.

${ }^{1}$ Enfermeira. Doutora em Enfermagem. Diretora do Departamento de Pesquisa e Projetos Estratégicos do Instituto Municipal de Pesquisas, Administração e Recursos Humanos de Fortaleza - Ceará.

${ }^{2}$ Enfermeira. Mestranda pelo Programa de Pós-Graduação Cuidados Clínicos em Enfermagem e Saúde da Universidade Estadual do Ceará-UECE. Membro do Grupo de Pesquisa Educação, Enfermagem, Saúde e Sociedade.

${ }^{3}$ Enfermeira. Mestranda pelo Programa de Pós-Graduação em Enfermagem da UFC.

${ }^{4}$ Enfermeira Assistencial do Sistema de Saúde Hapvida.

${ }^{5}$ Enfermeira Assistencial do Hospital Distrital Edmilson Barros de Oliveira Messejana. Especializanda em Auditoria em Serviços de Saúde Pública e Privada pelo Centro de Qualificação em Ensino Profissional.

${ }^{6}$ Enfermeira. Doutora em Enfermagem. Professora do Curso de Graduação e do Programa de Pós-Graduação em Enfermagem da UFC. Membro do Grupo de Pesquisa Políticas e Práticas de Saúde.

Autor correspondente:

Recebido: 23/07/2012

Cíntia Lira Borges

Aprovado: 02/10/2012

Universidade Estadual do Ceará

Av. Filomeno Gomes, 860 - 60010-281 - Fortaleza-CE-Brasil

E-mail: cintialiraborges@yahoo.com.br 


\section{INTRODUÇÃO}

O envelhecimento populacional gera uma série de questões cruciais para gestores e pesquisadores dos sistemas de saúde e das repercussões desse fenômeno na sociedade como um todo, especialmente num contexto de acentuada desigualdade social, pobreza e fragilidade das instituições ${ }^{(1)}$. Um dos resultados dessa dinâmica é a maior procura dos idosos por serviços de saúde. Assim sendo, as internações hospitalares são mais frequentes e o tempo de ocupação do leito durante a velhice é maior quando comparado a outras faixas etárias ${ }^{(2)}$. As causas de internação são percebidas também na prática diária durante a assistência domiciliar a idosos acamados após a alta hospitalar.

A úlcera por pressão (UPP) é uma das consequências concretas encontradas nesse cenário e o seu aparecimento independe da condição financeira do idoso. No cuidado a essa afecção, devem ser consideradas as doenças de base, o estado nutricional, o estado emocional, o comprometimento neurológico e a mobilidade prejudicada ${ }^{(3)}$.

Para idosos acometidos por UPP, ou com risco de adquirí-la, existem protocolos padronizados e normatizados para prevenção e cuidado. De acordo com a literatura ${ }^{(4-5)}$ esses protocolos fornecem ampla visão das reais necessidades do paciente, dando subsídios para o enfermeiro promover a sistematização da assistência com intervenções individualizadas e efetivas, contribuindo para tomada de decisões, reduzindo a incidência de UPP, promovendo a economia de equipamentos e evitando sobrecarga da equipe de enfermagem ${ }^{(6)}$.

Neste estudo, foi utilizado o protocolo prático do padrão de enfermagem para a prevenção de UPP, desenvolvido por enfermeiras como parte do projeto do Sistema de Melhoria nos Cuidados de Enfermagem para Idosos (NICHE- Hartford Institute for Geriatric Nursing). Esse projeto proporciona informações sobre saúde para instituições que promovem cuidados com base em pesquisas para populações em envelhecimento ${ }^{(7)}$.

Diante do exposto, o presente estudo tem por objetivo verificar a influência da aplicação de um protocolo de enfermagem para a prevenção de úlcera por pressão em idosos acamados no domicílio.

\section{A TRAJETÓRIA PERCORRIDA E O CONTEX- TO DA EXPERIÊNCIA}

Para adequare validar um protocolo de enfermagem de prevenção de UPP optou-se pelo estudo com delineamento experimental randomizado. Para dar-se início a este procedimento foi obtido o consentimento daautora do protocolo ${ }^{(7)}$.
Este estudo dividiu-se em duas etapas: a validação de conteúdo por enfermeiras especialistas em estomaterapia e a validação clínica com pacientes idosos com risco de desenvolver UPP. O protocolo foi aplicado por cuidadores domiciliares durante 30 dias, com a supervisão semanal do enfermeiro, tendo como metas principais a prevenção de UPP e o reconhecimento precoce do seu desenvolvimento.

O protocolo ${ }^{(7)}$ inclui fatores de risco intrínsecos e extrínsecos, a Escala de Braden ${ }^{(8)}$, a avaliação dos resultados esperados em relação ao paciente/cuidador/ enfermagem durante o período em que o idoso estiver sem deambular, bem como a monitorização da eficácia das intervenções preventivas.

A coleta de dados foi realizada no período de junho a agosto de 2010; a população do estudo foi composta de 520 idosos internados em cinco hospitais do Estado do Ceará. Quatro hospitais são de grande porte, sendo dois vinculados à rede estadual, servindo de referência para todo o Estado, um à rede municipal e o outro à rede privada. Os dois hospitais restantes são de médio porte e atendem em âmbito municipal.

Foram recrutados 40 idosos participantes que receberam alta no período do estudo e que obedeceram aos critérios de elegibilidade: idoso sem UPP, classificado de acordo com a Escala preditiva de Braden, incapaz de deambular, residente na cidade de Fortaleza ou região metropolitana, bem como aceitação do cuidado, destreza e habilidade por parte do cuidador. Os critérios de exclusão foram: cuidador analfabeto e avaliação insatisfatória do cuidador com relação à cognição.

Para a randomização, cada um dos participantes apresentou probabilidade igual para integrar o grupo experimental (A) e grupo controle (B). Para isso, foi estabelecida uma lista numerada de 1 a 40 , contendo o nome da instituição hospitalar, o nome do participante, seu endereço e telefone. O primeiro participant recrutado integrou o grupo $\mathrm{A}$, o segundo a lista do grupo $\mathrm{B}$, e assim sucessivamente.

Ao grupo A ( $\mathrm{n}=20)$ foi aplicado o protocolo de prevenção de UPP, em contrapartida, ao grupo B $(n=20)$ não foi aplicado tal protocolo. Ambos os grupos foram visitados semanalmente, sendo o grupo A para supervisão, avaliação da aplicação do protocolo pelo cuidador e da Escala de Braden; e o grupo B para a avaliação através da escala preditiva já citada.

Esta pesquisa foi aprovada pelo Comitê de Ética em Pesquisa do Hospital de Messejana (processo n. 706/10) e pelo Comitê de Ética em Pesquisa do Instituto Dr. José Frota (processo n. 2128/10). 


\section{ESTRATÉGIAS ELABORADAS PARA O DE- SENVOLVIMENTO DA PESQUISA}

\section{Processo de validação de conteúdo}

Nessa etapa ocorreu a avaliação da ferramenta de medição, através do consenso entre especialistas da área, no intuito de avaliar se o instrumento possui itens apropriados para medir o constructo específico e cobrir seu domínio ${ }^{(9)}$. Destarte, o protocolo foi avaliado por 16 enfermeiras especialistas em estomaterapia indicados pela Sociedade Brasileira de Estomaterapia, Sessão Ceará com, no mínimo, dois anos de experiência clínica na área. Entre os especialistas, três eram mestres em Enfermagem, com pesquisas relativas à temática feridas, um tinha o título de especialista em Gerontologia e outro era livre docente da área de Enfermagem.

Após esta seleção, foram agendados dois encontros para apresentação e adequação do protocolo, já traduzido para o português ${ }^{(7)}$. Essa adequação cultural se refere à realidade condizente ao estudo.

A técnica de coleta de dados utilizada foi o grupo focal a qual consiste em uma vivência de aproximação que permite a interação entre os participantes e o pesquisador, que objetiva colher os dados a partir da discussão focada em um tema particular ${ }^{(10)}$. Os encontros aconteceram na Associação Brasileira de Enfermagem, Sessão Ceará. No primeiro encontro, onde estiveram presentes sete especialistas, foram explicitados os objetivos da pesquisa e foi realizada a leitura do protocolo. Em seguida, foram concedidos alguns minutos para que o comitê de juízes de estomaterapeutas julgasse cada item, individualmente, atentando para relevância e necessidade de incorporá-lo, ou não, ao protocolo.

Em um segundo encontro, foi apresentado para o comitê, o protocolo resultante da consolidação das informações e dos julgamentos do primeiro encontro. Mediante o exposto foram realizadas alterações adicionais para, em seguida, obter o produto final do protocolo. Os encontros duraram em média uma hora e trinta minutos e os especialistas que foram impossibilitados a comparecer aos encontros enviaram sugestões por meio eletrônico.

\section{Processo de validação clínica}

\section{Seleção dos enfermeiros colaboradores}

Para validação clínica do protocolo no domicílio, foram convidadas 12 enfermeiras, da Estratégia Saúde da Família (ESF) do Município de Fortaleza-Ceará, para participarem de dois treinamentos.

O primeiro treinamento teve o intuito de promover a interação entre a equipe de enfermeiros da ESF e a pesquisadora, com vistas à compreensão do estudo, apresentação do projeto, do protocolo e da Escala preditiva de Braden. Das 12 enfermeiras convidadas, apenas duas compareceram ao treinamento, sendo necessário um novo encontro, com duração de quatro horas e teve como facilitadoras a pesquisadora-coordenadora e uma enfermeira especialista em estomaterapia.

No final, apenas cinco profissionais representantes de três Secretarias Executivas Regionais do município, as quais eram responsáveis pela execução dos serviços de saúde, educação, infraestrutura e habitacionais, dentre outros, participaram e aceitaram supervisionar a aplicabilidade do protocolo pelo cuidador.

Participação de acadêmicas de Enfermagem e inclusão de outros colaboradores no recrutamento aos idosos

A proposta inicial para o recrutamento dos idosos era ser por meio do contato telefônico da enfermeira da instituição com a pesquisadora, por ocasião da alta hospitalar do idoso. Tendo em vista as dificuldades referidas pelas enfermeiras em identificar os idosos no momento da alta e os critérios de elegibilidade, foi necessário alterar a estratégia de recrutamento. Para isso, foram convidadas acadêmicas do Curso de Enfermagem da Universidade Federal do Ceará, integrantes do Grupo de Pesquisa Idoso em Situação de Saúde e Doença nos Contextos Institucional, Social e Familiar para auxiliar na busca ativa dos participantes nas instituições selecionadas.

Houve uma reunião com um grupo de seis acadêmicas para compreensão do projeto e para a divisão das atividades entre as instituições hospitalares participantes, e identificção da população idosa através dos censos hospitalares e/ou diretamente nas enfermarias. Após os idosos atenderem aos critérios de elegibilidade, as acadêmicas foram orientadas a fornecer o contato telefônico da pesquisadora para que os acompanhantes/ cuidadores ligassem para informar a alta, no máximo até 24 horas após esta, a fim de que os idosos pudessem receber a primeira visita ainda na instituição ou no domicílio.

Também foram convidados agentes administrativos, que faziam parte do processo de alta do paciente, que ficaram cientes dos propósitos da pesquisa e contribuíram para a busca dos idosos participantes. 


\section{Participação do cuidador/família}

Após a etapa de recrutamento do idoso foram realizadas as visitas domiciliares. Muitas dificuldades foram enfrentadas nesse momento, entre elas a dificuldade para localização do endereço e dos contatos telefônicos, uma vez que muitos foram informados incorretamente, e frequentemente, o idoso retornava para casa dos filhos e/ou outros parentes, em vez do seu próprio domicílio.

No domicílio, o cuidador era informado acerca da importância do protocolo de UPP para a qualidade de vida do idoso, prevenção de novas internações e da morte. É importante ressaltar que houve boa adesão, interesse e envolvimento do cuidador/família no processo de prevenção e aplicação do protocolo. Os cuidadores foram informados sobre cada item do protocolo e como deveriam proceder durante a intervenção. A linguagem utilizada para abordagem, leitura e escrita do protocolo era simplificada, em concordância com a realidade do estudo, para que o cuidador não tivesse dúvidas ou dificuldades na execução das tarefas.

As atividades que deveriam ser realizadas pelo cuidador constituíam-se de 15 ações diárias, no prazo de 30 dias. Diariamente, os cuidadores deveriam marcar, em um espaço destinado no protocolo, se realizaram a ação: 'conforme protocolo' (CP), 'fiz parcialmente' (P), 'Não fiz/Por quê?' (NF). Além disso, poderiam escrever observações acerca do cuidado realizado.

As ações relacionavam-se à inspeção da pele durante o banho; limpeza da pele com água morna e sabão neutro, conforme a troca de fraldas a cada três horas; não massagear as proeminências ósseas, principalmente em áreas avermelhadas; hidratação do corpo com 100 mililitros de ácidos graxos essenciais misturado com 200 mililitros de hidratante, aplicando três vezes ao dia; na transferência do idoso de um local para o outro, elevá-lo e não arrastá-lo; virar o paciente a cada duas horas segundo a escala de horários; elevar o tornozelo do paciente, evitando contato com o colchão; utilizar posição lateral de $30^{\circ}$ na cama; estimular o paciente para executar atividades diárias para o autocuidado (pentear o cabelo, alimentação, higiene), entre outras.

O resultado do uso do protocolo foi positivo, com a incidência de UPP no grupo experimental de dois casos $(10 \%)$ e no grupo controle de 7 (35\%) ( $p=0,063)$. O grupo controle apresentou risco relativo para úlcera de 3,5 em relação ao grupo experimental. Esse valor mostra significância estatística ao nível de $10 \%$ pelo Teste Exato de Fisher unilateral. Os participantes que apresentaram UPP foram acompanhados por mais 30 dias.

\section{CONSIDERAÇÕES FINAIS}

Destaca-se que o construir/percurso desse estudo foi uma experiência oportuna de observação/ vivência da realidade dos idosos moradores da comunidade, muitos deles em precárias condições de vida e com baixo poder aquisitivo, expostos a fatores adversos de saúde, como fragilidades, vulnerabilidades, incapacidades e deficits nutricionais. Além disso, contribuiu para a percepção de que o trabalho na atenção primária deve ser valorizado e priorizado para melhores cuidados em saúde e para implantação de medidas preventivas, principalmente no que tange aspectos relacionados ao envelhecimento.

No contexto dessa vivência, também foi de extrema importância a construção de uma rede social de apoio, com a participação de diversos profissionais e estudantes, que contribuiu para a concretização do estudo.

Concluiu-se que medidas básicas e adequadas de prevenção podem impedir o aparecimento de UPP em idosos acamados ou em processo de hospitalização. A eficácia aumenta quando ocorre o uso de protocolo que dê continuidade ao trabalho preventivo no domicílio e auxilie os cuidadores durante a assistência ao idoso.

Observou-se ainda que a prevenção de UPP é um dos maiores desafios no cuidado de idosos acamados no domicílio. Dessa forma, compreende-se que, para prestar assistência integral e com qualidade, devem ser consideradas as dificuldades relacionadas aos idosos, à própria condição social e o vínculo do idoso com sua família como provedora de condições para a prestação de cuidados.

\section{REFERÊNCIAS}

1. Carvalho JAM, Rodriguez-Wong LL. A transição da estrutura da população brasileira na primeira metade do século XXI. Cad. Saúde Pública. 2008;24(3):597-605.

2. Veras R. Envelhecimento populacional contemporâneo: demandas, desafios e inovações. Rev. Saúde Pública. 2009;43(3):548-54.

3. Carraro TE, Sebold LF, Kempfer SS, Frello AT, Bernardi MC. Ensinar-aprender a cuidar de feridas: experiência de enfermeiras estagiárias docentes. Cogitare enferm. 2012;17(1):158-61. 
4. Edwards M. The rationale for the use risk calculators in pressure sore prevention, and the evidence of the reliability and validity of published scales. J. Adv. Nurs. 1994;20(2):288-96.

5. Medeiros ABF, Lopes CHAF, Jorge MSB. Análise da prevenção e tratamento das úlceras por pressão propostos por enfermeiros. Rev Esc Enferm. USP. 2009;43(1):223-8.

6. Chaves LM, Grypdonck MH, Defloor T. Pressure ulcer prevention in homecare: do Dutch homecare agencies have an evidence-based pressure ulcer protocol? J. Wound Ostomy Continence Nurs. 2006;33(3):273-80.

7. Ayello EA. Prevenção de úlceras à pressão e marcas na pele. In: Mezey MD, Fulmer T, Abraham I, Zwicker D. Protocolos em enfermagem geriátrica. São Paulo: Andrei; 2006. p. 177-95.

8. Paranhos WY, Santos VLCG. Avaliação de risco para úlceras de pressão por meio da escala de Braden, na língua portuguesa. Rev Esc Enferm. USP. 1999;(33):191204.

9. Polit DF, Beck CT. Fundamentos de pesquisa em enfermagem: avaliação de evidências para a prática da enfermagem. $7^{\text {a }}$ ed. Porto Alegre: Artmed; 2011.

10. Ressel LB, Beck CLC, Gualda DMR, Hoffmann IC, Silva RM, Sehnem GD. O uso do grupo focal em pesquisa qualitativa. Texto Contexto Enferm. 2008;17(4):779-86. 